\title{
THE POSSIBILITY OF USING SIGN AND SYMBOLIC TOOLS IN THE DEVELOPMENT OF MOTOR SKILLS BY BEGINNING SOCCER PLAYERS
}

\author{
Aleksander N. Veraksa, \\ Aleksandra E. Gorovaya, \\ Sergey V. Leonov \\ Lomonosov Moscow State University \\ Moscow, Russia \\ Aleksander K. Pashenko, \\ Valeriy V. Fedorov \\ Moscow State University of Psychology and Education \\ Moscow, Russia
}

This article analyzes the use of iconic and symbolic tools by young athletes in the development of concrete motor skills. The study involved 22 young athletes, aged 5-6 years, attending a soccer school in Moscow, Russia. The methodological procedure included using specifically designed mini-movies, which were short video clips employing different sign and symbolic tools for mastering alternate dribbling using the inner and outer side of the foot and the subsequent kick of the ball toward the net. The results showed the effectiveness of these tools when working with young soccer players. Those athletes who used metaphors as one of the tools for mastering motor skills exhibited better movement development than did the athletes who used sign tools.

Keywords: sport, sport psychology, motor skill, sign, symbol, metaphor, image.

A number of works devoted to the development of motor skills demonstrate the effectiveness of using sign and symbolic tools (Palmer, 1992; Garza \& Felts, 1998; and others). However, because of the differences involved in using sign and symbolic tools, they were shown to be not quite efficient (Veraksa, 2009). Therefore, we have turned to the study of metaphor as this form of iconic and symbolic expression is closer to the symbol on the "sign-symbol" axis (Losev, 1995). 
In fact, metaphor follows the principle of building a symbol, with the only difference being that the content of a metaphor, as opposed to a symbol, refers to a definite reality - that is, to the value that lies behind the metaphor.

Thus, in describing poetic language, which is without doubt a striking example of the use of metaphors, Ricoeur (1990) notes that it "creates a kind of 'fusion' of meaning and sensations - that is, sensory perception - which distinguishes it from nonpoetic language; in such language, because of the arbitrariness and conventionality of the sign, the sense is extensively "deprived' [of] the sensor component... the language, instead of being just something on the way to the reality, itself is material like marble for the sculptor" (p. 441). In this sense metaphor is presented as a single instance of character and image.

Metaphor, like symbol, can be converted into a sign when it is used repeatedly; the result is a fusion of sign and image in which the meaning begins to dominate. Thus, Wheelwright (1990, p. 91) wrote that "in modern everyday language there are many words and expressions which have arisen from the early 'light' metaphor [that] can be adduced to [be] the phenomena of the mental plane. They are to clarify, illuminate, illustrate a clear, bright image, etc. In general, all these words have ceased to function as active metaphors and have lost any power, becoming the current coin."

As Goodman remarked (1976), metaphor involves mapping one kind of object in other domains. Gombrich provides the following example, in which he uses words that are not quite meaningful for us, ping and pong: "If these words would have been all that we have, and we had to call an elephant and the cat, who would be ping and who would be [pong]? I think the answer is obvious. Or hot soup and ice cream. For me, at least, ice cream would mean ping, and the soup will [be] pong" (1960, p. 370).

Gombrich points out that the words ping and pong previously would not have had any meaningful value as they did not denote anything.

Thus, the classification built on these two words is not based on past signification experience but on what these words denote now. Mapping based on these words occurs through analyzing their external sound shell, which does not lead to symbolic understanding of them but to metaphorical signification: ping - lightweight, fast, cool; pong - heavy, slow, hot. According to Aragno (2009), such comparisons give rise to novel and inconventional metaphors. 


\section{The Emotional and Cognitive Function of Metaphor in Sport: Recent Research}

In sport psychology, metaphor is considered an effective tool when working with athletes (Jones, 2003). In such cases, the emotional function of metaphor comes to the fore. For example, Hanin's research shows that metaphor is used spontaneously by athletes to express their emotional state (Hanin \& Stambulova, 2002).

Moreover, sometimes only metaphorical language is available for athletes to describe emotions they are experiencing (Ruiz \& Hanin, 2004). Lindsay, Thomas, and Douglas (2010) describe a model of modified metaphors by means of which athletes describe their condition. The model involves five stages: the definition of metaphor that is used by an athlete; a preliminary analysis of metaphor aimed at identifying the image appearing in a referent situation; the expansion of metaphorical descriptions (designation of the image content, the time when it reveals feelings that this image recalls, and so forth); the modification of metaphors (by means of changing the image); the use of a new metaphor in a real-life situation. According to the authors, modification of the metaphor entails changing the athlete's attitudes toward the situation.

Let us consider the cognitive function of metaphor, which is our particular area of interest. According to Modell, at first, "metaphor was thought to be a trope, a figure of speech, a kind of verbal slight of hand in which meaning is transferred by means of analogy." But "metaphor not only transfers meaning between different domains, but by means of novel recombinations metaphor can transform meaning" (2009, p. 1). Psychological science has accumulated vast experience in the use of metaphor as an effective means of developing and structuring various contents (for example, Cameron, 2002). In sport, metaphor as an agent of cognition is used to separate implicit learning from explicit learning. "When individuals accumulate knowledge passively and no conscious analytic strategies are used, the learning process is considered implicit. ...Implicit learning is passive[,] without perceived use of analytical strategies... Knowledge acquired through explicit learning is accessible to consciousness and can be articulated" (Liao \& Masters, 2001, p. 307).

For example, it has been shown that professional batters have limited knowledge of the kinematics of proper motions, so close attention to them reduces the success of their actions (Gray, 2004). 
Implicit learning does not involve loading the individual's working memory, so its success has no correlation with IQ (as opposed to explicit learning) (for example, Reber, Wlkenfels, \& Herstadt, 1991). Masters (1992) has proposed that the reduced effectiveness of performing any sport activity as a result of stress can be explained by appeal to explicit knowledge. If the athlete has automatically achieved performance as a result of explicit instruction, later, in a stressful situation, the automatic skill fails, and the participant requires resources to reproduce the explicit knowledge.

Therefore, according to Masters (1992), an effective way to counteract stress factors is by minimizing explicit knowledge and therefore explicit teaching.

A good example of the use of metaphor as a means of implicit learning can be found in the research by Poolton, Masters, and Maxwell (2006), in which the participants were taught to play table tennis. They were given the task of hitting the ball with the paddle so that the ball kicked off a marked surface of the tennis table.

The participants were divided into two groups. In the first group instruction in how to hold the paddle was given in detailed form through the explanation of several rules (explicit learning).

The participants in the second group (who were learning through metaphor) were given the following instruction: move the paddle as if it were going up a hill.

No comments followed the instructions to both groups. After the same number of preliminary tests, the participants were required to solve two types of tasks. The easy task was to hit balls of two colors: the white one had to go to the right part of the table, and the yellow one had to go to the left part of the table. The complex task was similar to the easy one with only one difference: after every two balls the color of the balls and the part of the table they were required to be in were reversed.

For the easy assignment no differences were found between the two groups of participants. In fulfilling the complex task, the participants who were instructed by using an analogy proved to be much more successful. The authors believe that the use of metaphor helped to improve the results in the complex task because the image (as opposed to the verbal instructions) did not activate the working memory - that is, phonemic coding was omitted. 
The research paradigm of increasing the cognitive complexity of the problem and studying the resulting effectiveness in performing the motor skill at first glance may seem artificial, devoid of the "refined" reality of sport performance. However, as practical experience has shown, the performances of highly achieving athletes depend on the ability to consider multiple factors and to respond to a rapidly changing environment.

Liao and Masters (2001) also examined the possibility of using implicit learning to teach master strokes in table tennis. The participants were set the task of directing the ball onto the marked surface of a tennis table using a strong top spin.

The participants earned points if they hit the ball properly and reached the marked table areas. The participants were students who had no previous experience of learning to play table tennis and who were not practicing frequently. The participants were divided into three groups: those who were taught by analogy (in which the participants were instructed to draw a right triangle with the paddle and to hit the ball when the paddle was moving along the imaginary hypotenuse); those who were taught using explicit instruction (in which they were taught 12 basic skills); those who were taught using implicit learning (in which they were not given any instructions on how to hit but were given the task of pronouncing the letters of the alphabet while making hand movements at a speed of one letter per second).

After completing the task ( 6 episodes of 50 hits each) the participants had to fill out a questionnaire in which they could assess the knowledge that they gained during the experiment-that is, the knowledge that they used or developed while performing the task. The results were similar for the group that was taught by analogy and the group that was taught using implicit learning - participants in both groups were able to formulate one rule on average - while the participants in the explicit-learning group on average described more than six rules each.

All participants were also asked to assess their use of confidencebuilding skills. Only in the explicit-learning group did this index correlate with the actual results. The authors suggested that the weak awareness of the process of skill mastering in the analogy group led to the participants in this group being more resistant to stresses than were the participants in the explicit-learning group.

To verify this hypothesis the following experiment was carried out. The participants in the analogy group and in the explicit-learning group 
were subjected to stress: after 6 episodes of 50 hits the participants reported that their results were worse than the experimenter expected.

Then the participants were asked to make greater efforts to achieve better results. The stress effect had a positive impact on the analogy group, whose results significantly increased, while in the explicit-learning group results worsened. At the same time, "in both experiments, the analogy learning group showed learning curves similar to the explicit learning group" (Liao \& Masters, 2001, p. 317). According to the authors, these results support the hypothesis that the less the perceived knowledge is, the less it suffers from stress.

Similar results were obtained by Maxwell, Masters, and Eves (2000) in teaching participants golf strokes. For the explicit instruction a strong motor image was developed in the participants solely through frequent repetition; in a complex situation such learning requires cognitive restructuring, which eventually reduces the efficiency of movement. When a metaphor is proposed for learning the movement, a participant interprets the image based on the specific motor task and fills it with appropriate designates implied by the situation.

\section{Age-Related Aspects of the Use of Metaphor}

In the literature the age at which individuals acquire metaphors and can manipulate them freely is extensively discussed (Knowles\& Moon, 2006).

The research shows that already at early-preschool age children can understand metaphors. Gentner (1988) identifies three types of metaphors: object mappings (which are associated with specific properties, such as "the sun is like an orange" - both have an orange color and round shape); relations mappings (which are related to structural characteristics, such as "a tire is like a shoe" - both objects can go a long way, and both are in contact with the surface); and double metaphors (which have both common external features and common structural organization, such as "a stalk is like a straw for the plant" - both objects are of cylindrical shape, and both perform the function of liquid absorption). Preschool-age children are able to use metaphors of all these types.

Similarly, Pramling and Pramling Samuelsson (2009) provide evidence that preschool children spontaneously resort to metaphor to describe various situations. 
Within the framework of psychoanalysis, as well as within the framework of phylogeny and ontogeny, there is a perception that metaphor occurs early as a means of communication via body language (Modell, 2003). In this regard, we can assume that motion will be the most accessible material for children, so it can be mastered by means of metaphors. In research by Sawada, Mori, and Ishii (2002) on teaching movement to children through the use of metaphor, the participants were 30 children aged 5-6 years who were divided into two groups: in the first group each child alone watched a sequence of movements performed by the teacher, who first named and then showed them one after another ("sit down stand up - jump - bend - fall"); in the second group each child alone watched as the teacher performed the same sequence of movements but used metaphors for naming them ("flower in the bud - soon it will be revealed - butterfly flutters out-it sleeps on a flower - it falls from the flower"). Children in the second group were able to remember the sequence of movements and reproduce the sequence significantly more easily than children in the first group.

Gardner, Winner, Bechhofer, and Wolf (1978) found that preschoolage children are sensitive to the use of metaphor, but at school age this sensitivity is greatly reduced, and it appears again in adolescence.

This pattern may have several explanations. If we consider metaphor not only as an instrument of the content structure based on analogy but also as having a marked emotional component, it is obvious that for a spontaneous "use" of metaphor in different age groups the conditions are not equal.

During the early stages of schooling the preference for the development of formal logical thinking reduces the manifestation of imagination and creative thinking in children. In our research (Veraksa \& Gorovaya, 2011), young children were likely to use emotionally charged images in sporting activities, where these images play a motivational role; in preadolescents cognitive images dominate and reflect the structure of sport activity.

Most research we have examined shows the effectiveness of metaphor as a tool for acquiring physical skills. However, it should be emphasized again that despite the fact that a metaphor is very close to a symbol (as compared with an emblem, allegory, or other signs and symbolic tools), metaphors vary significantly based on how "transparent" the analogy is. For example, the image may be schematic (as in the description of pad- 
dle movements in table tennis), or the image may be not only cognitive but also emotional (as in the case of an image of oneself as a chrysalis); this self- image is less amenable to being "rendered" in the language of a real-life situation.

As we have observed previously (Veraksa \& Gorovaya, 2011), given the transition from motivational images in younger age groups to cognitive images in more mature groups, we should expect differences in metaphor usage between children and adults not only according to the logical structure of metaphors (as proposed by Gentner) but also according to the degree of symbolism.

At the same time, interviews conducted with athletes and coaches in various sports show that metaphors used in adolescence have a mixed character. For example, when divers perform the "disclosure" movement in the jump right before entering the water, they are mimicking the condition described by "a bow string." During this "opening" movement in diving, a number of athletes also use the triangle metaphor, for they notice that to enter into the water appropriately they first need to "create a body triangle." In addition, athletes use metaphors that embody the sense of a holistic jump rather than those that describe particular elements such as "stretched like a string" or "I am mighty."

\section{Study}

The accounts mentioned above allowed us to devise a study to ascertain whether metaphor is an effective tool for developing sport skills in young children.

\section{Participants}

The participants were young athletes, aged 5-6 years $(\mathrm{N}=22, \mathrm{M}=$ 5.7 years), attending the Chertanovo Sport School in Moscow, Russia. In the main part of the study, the participants were divided into two groups so that ages and general physical ability were the same in both groups; physical ability was measured by the development of characteristics important in soccer such as speed, agility, and endurance. The main difference in the training of the two groups of players consisted in using either sign tools or metaphors to develop certain movements. The group of participants in which the sign tools were used consisted of 10 players, and the group in which metaphors were used contained 12 players. 
The procedure for implementing the intervention was similar for both groups of participants. In the course of the research, all athletes continued their usual physical exercises in the same way as before the intervention.

\section{Method}

In the preliminary stage of the experiment, individual, semistructured coach interviews were conducted; the interviews were directed at providing an understanding of the specific features of the way athletes of this age play soccer. They also included information about the general physical preparation of each athlete, the features of the training process, and the motivation of the athletes. The central motor skill to be developed was alternate dribbling by the outer and inner side of one foot, ending with a strike of the ball directed to the net.

\section{Performance Rates}

Monitoring the success of the training in developing players' motor skills was conducted by controlling changes in individual indicators of dribbling.

Monitoring was carried out by watching videos of the athletes' movements.

The choice of motor skill as an object of research was determined through consultations with the coaches; the choice takes into account both the age characteristics of this group of athletes and their physical ability.

The assessment of successful performance was carried out by looking at a number of indicators that reflect the constituent elements of the movement: dribbling, directing the ball, and finally striking the ball. In addition, the results of coach interviews were an additional integral indicator of the development of the movement as a whole.

Given the need to reduce the influence of subjective factors, the procedure to evaluate the success of mastering the movement was built on expert estimates. For this evaluation, the role of specialists was performed by four experts, professional soccer coaches.

\section{Sign and Symbolic Tools}

The main methodological procedures used in the intervention were mini-movies modeled specifically for each group of athletes (those who 
were learning through sign tools and those who were learning by using metaphors). These short videos (30 seconds each) used a variety of signs and symbolic tools of alternate dribbling by the outer and inner side of one foot, ending with a strike of the ball toward the net.

Both video clips had identical starting and ending camera shots, which showed a coach performing the movement. The central fragments of the video clips were different. The first of them, for the athletes in the sign-tools group, was a drawing of a human being's performance of the movements (see Figure 1).

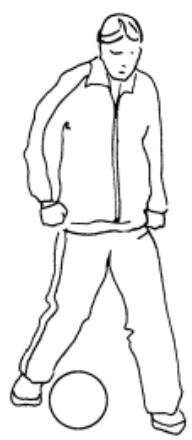

Figure 1. A scene from the mini-movie for the sign-tools group

In the second clip, designed for the metaphors group in the form of an animated cartoon, a symbolic image of the movement was presented (see Figure 2). In it a two-headed snake is making zigzag movements (similar to the coach's leg movements) and is chasing the mouse (the ball), which runs around a pebble (symbolizing the training rack) and hides in a burrow (reflecting the net) after the snake pushes it.
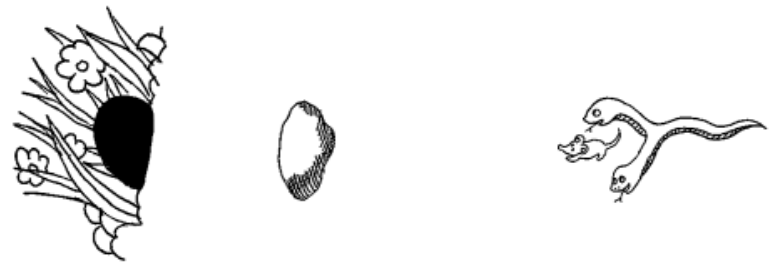

Figure 2. A scene from the mini-movie for the metaphors group 
The Appendix contains a shot-by-shot expansion of the central video-clip fragment for each group.

The athletes were individually invited to watch the mini-movie for their group once and then to answer questions that focused attention on the general plot of the movie and the actions performed by each of the characters.

\section{Procedure}

The preparation phase of the research was devoted to analyzing the athletes' abilities, specifying the motor skill that would be the object of analysis, and subsequently shooting the training mini-movies.

The main phase of the study was carried out during 4 weeks and included 12 individual sessions with the athletes. At each third meeting, each athlete's mastery of the skills was monitored by video.

All the novice players found the practical classes of great interest. Despite the fact that they had a significant number of meetings in which they saw the same video clips, they watched them with great pleasure.

During the experiment, four participants discontinued attending the sport school and therefore were not included in the data processing.

\section{Results and Discussion}

The monitoring data were assessed by expert analysis of indicators that reflected the constituent elements of the movement (dribbling, directing the ball, striking the ball). Moreover, in the coach interviews an integral indicator of the movement development was added; this indicator assessed an athlete's ability to integrate directing the ball, holding the direction, and striking the ball in a given time.

The results of the correlation analysis of success indicators confirm the value of the integral indicator. By using the Spearman correlation coefficient, we found statistically significant correlations at the $\mathrm{p}=0.01$ level for the integral indicator $(r=0.8)$, the direction of movement $(r=$ $0.6)$, and the kick $(\mathrm{r}=0,8)$.

The Mann-Whitney test, performed during the preliminary stage of the study, showed no statistically significant differences between these groups on the success indicators: dribbling $(\mathrm{p}=0.91)$, direction of movement $(\mathrm{p}=0.44)$, final kick $(\mathrm{p}=0.59)$, integral indicator of movement development $(\mathrm{p}=0.67)$. In other words, prior to the intervention the two groups were indeed equivalent with respect to these indicators. 
Application of the Wilcoxon test allowed us to compare the development of athletes' movement before the intervention and after its completion. Thus, for the sign-tools group, comparisons of dribbling the ball, directing the ball, and the final kick, as well as of the integral indicator of movement development, indicate no significant shifts $(\mathrm{p}=0.06, \mathrm{p}=0.71$, $\mathrm{p}=0.1$, and $\mathrm{p}=0.14$, respectively).

Assessment of the statistical significance of differences in the success of the metaphors-group athletes suggests the feasibility of using metaphors as a tool for developing motor skills. The results show the presence of significant shifts in all parameters (dribbling: $\mathrm{p}=0.046$, directing the ball: $\mathrm{p}=0.042$, integral indicator of movement development: $\mathrm{p}=0.046$ ) with the exception of striking the ball $(\mathrm{p}=0.07)$. A possible explanation may be that the symbolic content of the mini-movie for this group focused more attention on dribbling the ball and directing the movement of the ball (the image of the two-headed snake making zigzag movements) than on striking the ball (pushing the mouse to the burrow).

The results discount the possible correlation between the athletes' regular attendance at physical training and the successful performance of the indicators because there are no statistically significant correlations between them. This fact points once again to the role of sign and symbolic tools in attaining the performance indicators but is a kind of confirmation also of the principle of effective construction of the training process: quantity is not always transformed into quality; only a qualitative quantity is transformed into quality.

Additional consideration of the results of the study from the perspective of qualitative analysis is appropriate. Expert estimates allowed us to chart each athlete's success in performing the skill and to analyze the dynamics of developing a motor skill through the use of iconic and symbolic tools.

Each chart below groups athletes by initial level of physical ability (high, medium, and low) and by training group: $M$ (metaphors group) or $\mathrm{S}$ (sign-tools group). The performance of the athletes was assessed during the shooting of four videos (cuts) during the course of the training. Because of the difficulty of distinguishing an excessive number of similar curves on the charts, some of them are omitted.

The indicators and the range of assessment values for each of them were selected on the basis of the coaches' recommendations and took into account the athletes' ages and physical skills. 


\section{Dribbling}

In assessing dribbling the minimum value was 0 ; the maximum was 14. It was assumed that from 0 to 5 is a low level of ability, from 6 to 10 is a medium level of ability, from 11 to 14 is a high level of ability.

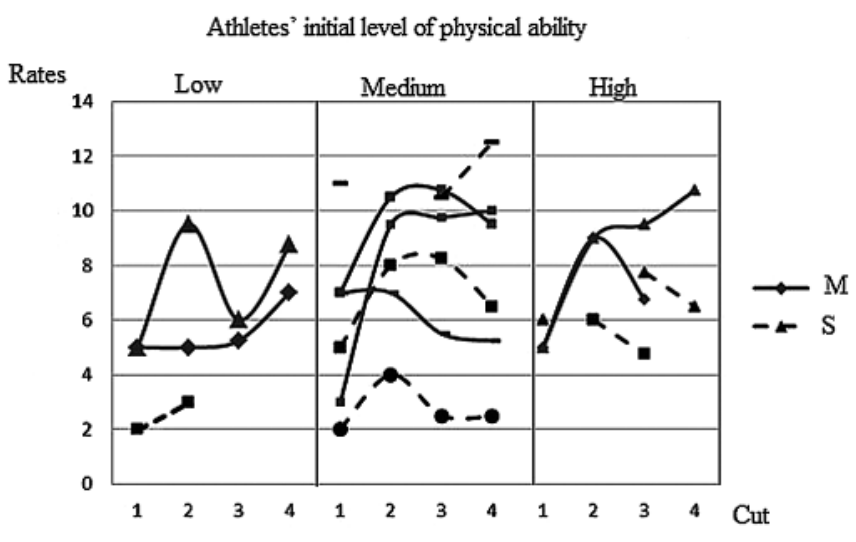

Chart 1. Dynamics of dribbling

Part 1 of this chart (athletes with a low initial physical-ability level) shows that the ability of all three athletes to dribble the ball at the first cut (video shooting) was below medium.

In the last cut (the fourth shooting), metaphors-group athletes demonstrated an increase to the medium level, and the sign-tools group showed only a slight increase in their rates and remained at a low level.

In addition we can see that between cuts 1 and 4 one athlete in the metaphors group slowly increased dribbling capabilities, and another player showed inconsistent results that changed from high to low to medium. Such impulsiveness seems to reflect the instability of the acquired skills.

Part 2 (athletes with a medium initial physical-ability level) shows that during the intermediate cuts all participants (independently of group affiliation) had unstable results (increase, then decrease, and vice versa).

At the same time, in comparison with the signs-tool group, the metaphors-group athletes demonstrated a clearer increase in their ability overall. 
Part 3 (athletes with a high initial physical-ability level) shows that by the fourth cut the metaphors-group participants showed better results than the sign-tools group.

In general we can say that athletes with different initial physical-ability levels in the metaphors group showed a more positive trend in ability development than athletes of the same levels in the sign-tools group.

\section{Directing the Movement of the Ball}

For assessing the ability to direct the ball, the minimum value was 2, and the maximum was 11. It was assumed that values from 2 to 4 show a low level of ability, from 5 to 8 show a medium level of ability, from 9 to 11 show a high level of ability.

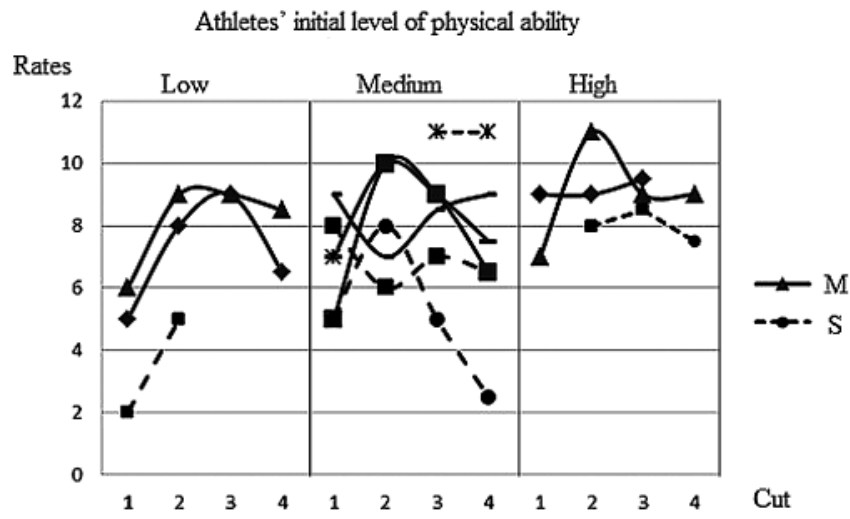

Chart 2. Dynamics of directing the movement of the ball

Part 1 of this chart (athletes with a low initial physical-ability level) shows that at the first cut all metaphors-group athletes demonstrated a medium level of ability. By the fourth cut they improved their performance and showed a more positive trend than the athletes of the signtools group in the ability to maintain the direction of the ball.

Part 2 (athletes with a medium initial physical-ability level) shows that all the metaphors-group athletes at the first cut demonstrated medium capability in maintaining ball direction. The second and third cuts show both rising and falling ability levels in both groups. By the fourth cut, almost all the metaphors-group participants demonstrated a medium level for this capability. 
Part 3 (athletes with a high initial physical-ability level) shows that at the first cut almost all athletes were close to the highest level. By the fourth cut metaphors-group athletes managed to improve their original figures or to keep them at the same level, while the sign-tools athletes showed a downward trend.

\section{Striking}

In assessing striking the ball to the net the minimum value was 0 , while the maximum was 12 . It was assumed that from 0 to 4 indicated a low ability level, from 5 to 9 showed a medium ability level, from 10 to 12 indicated a high ability level.

\section{Athletes' initial level of physical ability}

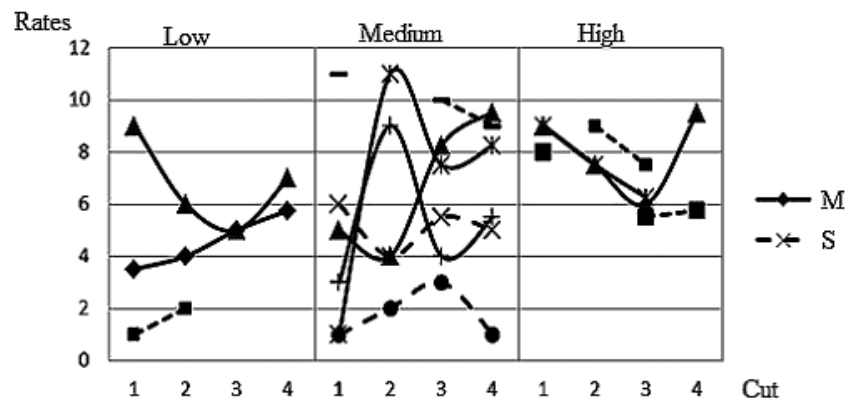

Chart 3. Dynamics of striking the ball

Part 1 of this chart (athletes with a low initial physical-ability level) shows that one metaphors-group participant displayed steady growth in ability from the lower level at the first cut to the medium level at the fourth cut. The remaining participants in the same group performed at the medium level at the first cut; their performance dropped at the second and third cuts, stopping in the middle of medium ability, and at the fourth cut it significantly increased.

Part 2 (athletes with a medium initial physical-level) shows that none of the athletes (regardless of group affiliation) showed a continuous increase in the technical performance of the strike, and impulsivity was observed. However, we can see that metaphors-group participants showed a more positive trend in kicking the ball than did the sign-tools group. 
Part 3 (athletes with a high initial physical-ability level) shows that the sign-tools group showed a decreasing trend in their ability to strike the ball. They performed at a medium level at the initial cuts, their results worsened, and at the final cuts they also showed a medium level of ability. Metaphors-group participants showed at the first cut the highest value of the medium level of ability, while at the fourth cut they either slightly improved their performance or remained at the medium level.

\section{Integrated Skill Mastery}

In assessing integral skill mastery the minimum value was 0 , while the maximum value was 10 . It was assumed that from 0 to 3 indicated a low level of ability, from 4 to 7 indicated a medium level of ability, from 8 to 10 indicated a high level.

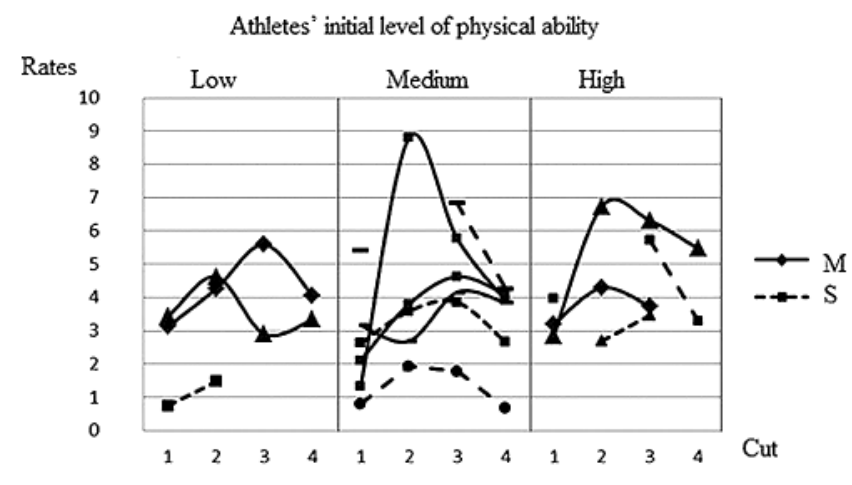

Chart 4. Dynamics of integrated skill mastery

Part 1 of this chart (athletes with a low initial physical-ability level) shows that the dynamics of the integral indicator of movement development by the athletes of the metaphors group had an impulsive character. Showing at the first cut a low level, at the fourth cut they improved their results.

Part 2 (athletes with a medium initial physical-ability level) shows that at the first cut all participants (regardless of group affiliation) possessed low levels of the integral indicator of movement develop- 
ment, while by the fourth cut those in the metaphor group had slightly improved their results. Growth varied from uniformly rising to impulsive.

Part 3 (athletes at a high level) shows that all metaphors-group participants managed either to improve their results or to remain within the medium level.

To generalize the qualitative analysis of motor-skill individual dynamics through the use of sign and symbolic tools, we find it necessary to reiterate some important findings.

We have shown that athletes in the metaphors group having a medium initial level of general physical ability exhibited a more positive trend in movement development than did athletes in the sign-tools group. At the same time, when comparing metaphors and sign-tools athletes with lower and higher levels of initial general physical ability, the observed trend is not obvious. This result appears to be partly explained by the specific features of soccer as a sport.

Inasmuch as the players need diversified training that focuses on a set of game-specific and general physical exercises, the initial level of general physical ability plays an important role in the development of specific motor skills.

Thus, athletes with initially higher levels of general physical ability may have a greater propensity to demonstrate good skill development than athletes with medium physical ability.

However, as reflected in the results attained, athletes with a low level of general physical ability demonstrated an increase in their ability to develop specific movements as their opportunities increased.

Another important point that needs to be focused on further is associated with the specific nature of motor-skill development in athletes. A number of the charts displayed a jagged form, which indicates the absence of a stable manifestation of the ability from cut to cut and the impulsive nature of skill development. Perhaps these results reflect the age-specific development of athletes' motor skills.

In addition, because a similar trend was typical of most athletes in the sign-tools group, it can be assumed that the use of metaphors as a tool can stabilize the process of mastering the movement. However, the issue remains open and clearly requires the careful development of further research. 


\section{Conclusion}

The research confirmed the hypothesis in general that signs and symbolic tools for learning motor skills can be effective in working with young players. Athletes who used metaphor as an additional tool to master motor skills exhibited a more positive trend in the development of alternate dribbling by the outer and inner side of one foot, ending with a kick of the ball on target, than did the athletes who used sign tools.

In addition, analysis of the individual dynamics of movement development showed that they have an individual character. If in some athletes the dynamic is rather even, in others it is of an impulsive or spasmodic character.

Athletes who used metaphors exhibited more positive trends than the athletes who used sign tools not only in general but also for a number of constituent elements of movement: the ability to learn dribbling (athletes with a high initial physical-ability level), the capacity to maintain the direction of the ball (athletes with a high initial physical-ability level), the ability to strike (athletes with a medium initial physical-ability level).

A number of athletes at the fourth cut showed a reduction in their results, despite the increase in the results at the second and third cuts.

In this regard, we cannot exclude the possibility of adverse effects on them (either external or internal). One possibility is that by the day of the fourth cut (May 16, 2011) fatigue had set; the end of the season and the closeness of holidays may have prevented them from benefiting fully from the training process.

\section{Acknowledgments}

The authors express their gratitude to A.V. Tsutskov, director of the Chertanovo Sport School, and N.L. Korovkin, coach of the team whose participants were born in 2004, for their help in organizing and conducting the research, and the authors thank as well all the athletes who participated in the research.

The research was supported by Grant of the President of the Russian Federation for state support of young Russian scientists № MK3455.2011.6. 


\section{References}

Aragno, A. (2009). Meaning's vessel: A metapsychological understanding of metaphor. Psychoanalytic Inquiry, 29, 30-47.

Cameron, L. (2002). Metaphors in the learning of science: A discourse focus. British Educational Research Journal, 5, 673-688.

Gardner, H., Winner, E., Bechhofer, R., \& Wolf, D. (1978). The development of figurative language. In K. Nelson (Ed.), Children's language, 1-37.

Garza, D., \& Feltz D. (1998). Effects of selected mental practice on performance, self-efficacy and competition confidence of figure skaters. Sport Psychologist, 12, 1-15.

Gentner, D. (1988). Metaphor as structure mapping: The relation shift. Child Development, 59, 47-59.

Gombrich, E.H. (1960). Art and illusion. New York: Pantheon.

Goodman, N. (1976). Languages of art: An approach to a theory of symbols. Indianapolis: Hackett.

Gray, R. (2004). Attending to the execution of a complex sensorimotor skill: Expertise differences, choking, and slumps. Journal of Experimental Psychology: Applied, 10, 42-54.

Hanin, Y., \& Stambulova, N. (2002). Metaphoric description of performance states: An application of the IZOF model. Sport Psychologist, 16, 396-415.

Jones, M.V. (2003). Controlling emotions in sport. Sport Psychologist, 17, 471486.

Knowles, M., \& Moon, R. (2006). Introducing metaphor. London: Routledge.

Liao, C.-M., \& Masters, R.S.W. (2001). Analogy learning: A means to implicit learning. Journal of Sport Sciences, 19, 307-319.

Lindsay, P., Thomas, O., \& Douglas, G. (2010). A framework to explore and transform client-generated metaphors in applied sport psychology. Sport Psychologist, 24, 97-112.

Losev, A. (1995). Problema simvola i realisticheskoe iskusstvo [Problem of symbol and realistic art]. Moscow: Iskusstvo.

Masters, R. (1992). Knowledge, (k)nerves and know-how: The role of explicit versus implicit knowledge in the breakdown of a complex motor skill under pressure. British Journal of Psychology, 83, 343-358.

Maxwell, J. P., Masters, R.S.W., \& Eves, F.F. (2000). From novice to no know-how: A longitudinal study of implicit motor learning. Journal of Sport Sciences, 13, 11101120.

Modell, A. (2003). Imagination and meaningful brain. Cambridge, MA: MIT Press. 
Modell, A. (2009). Metaphor - the bridge between feelings and knowledge. Psychoanalytic Inquiry, 29, 6-17.

Palmer, S. (1992). A comparison of mental practice techniques as applied to the developing competitive figure skater. Sport Psychologist, 6, 148-155.

Poolton, J.M., Masters, R.S.W., \& Maxwell, J.P. (2006). The influence of analogy learning on decision-making in table tennis: Evidence from behavioral data. Psychology of Sport and Exercise, 7, 677-688.

Pramling, N., \& Pramling Samuelsson, I. (2009). The prosaics of the figurative language in preschool: Some observations and suggestions for research. Early Child Development and Care, 3, 329-338.

Reber, A.S., Wlkenfels, F.F., \& Herstadt, R. (1991). Implicit and explicit learning: Individual differences and IQ. Journal of Experimental Psychology: Learning, Memory and Cognition, 17, 888-896.

Ricoeur, P. (1990). Living metaphor/ Theory of metaphor. Moscow: Progress.

Ruiz, M.C., \& Hanin, Y.L. (2004). Metaphoric description and individualized emotion profiling of performance states in top karate athletes. Journal of Applied Sport Psychology, 16, 258-273.

Sawada, M., Mori, S., \& Ishii, M. (2002). Effect of metaphorical verbal instruction on modeling of sequential dance skills by young children. Perceptual and Motor Skills, 95, 1097-1105.

Veraksa, A. (2009). Voprosi primenenija znakovogo I simvolicheskogo otrazhenija $\mathrm{v}$ sporte [The application of the sign and symbolic reflection in sports]. Kul'turno-istoricheskaya Psihologiya [Cultural-Historical Psychology], 1, 76-82.

Veraksa, A., \& Gorovaya, A. (2011). Effect of imagination on sport achievements of novice soccer players. In Y.P. Zinchenko \& V.F. Petrenko (Eds.), Psychology in Russia: State of the Art (Vol. 4, pp. 495-504). Moscow: Lomonosov Moscow State University; Russian Psychology Society.

Wheelwright, P. (1990). Metafora i real'nost' [Metaphor and reality]. In Teorija metafory [Theory of metaphor] (pp. 82-119). Moscow: Progress. 


\section{Appendix}

Shot-by-shot video images of the mini-movie for the sign-tools group:
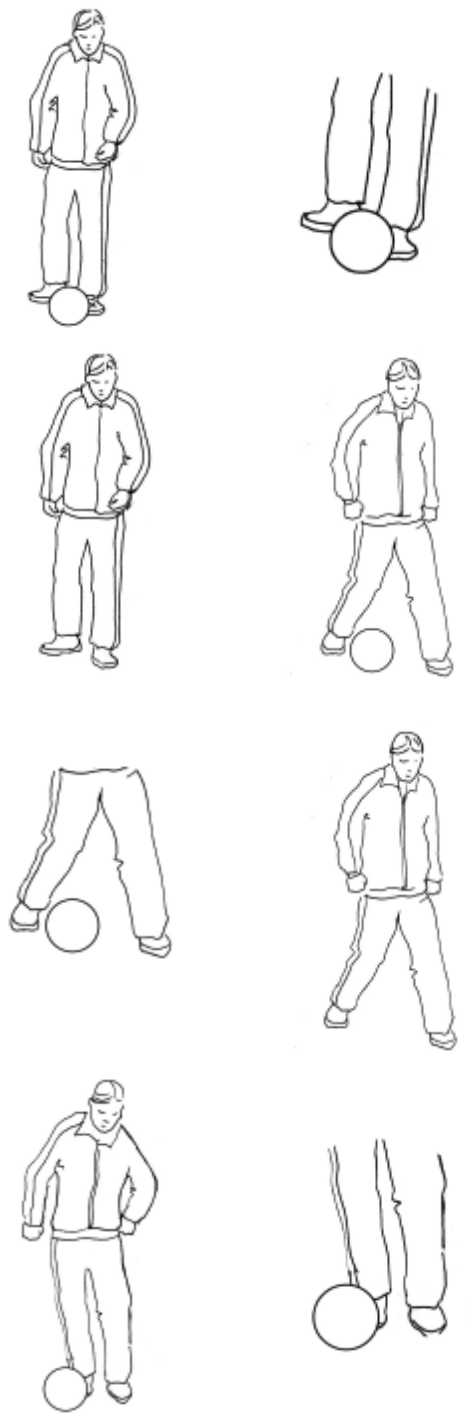


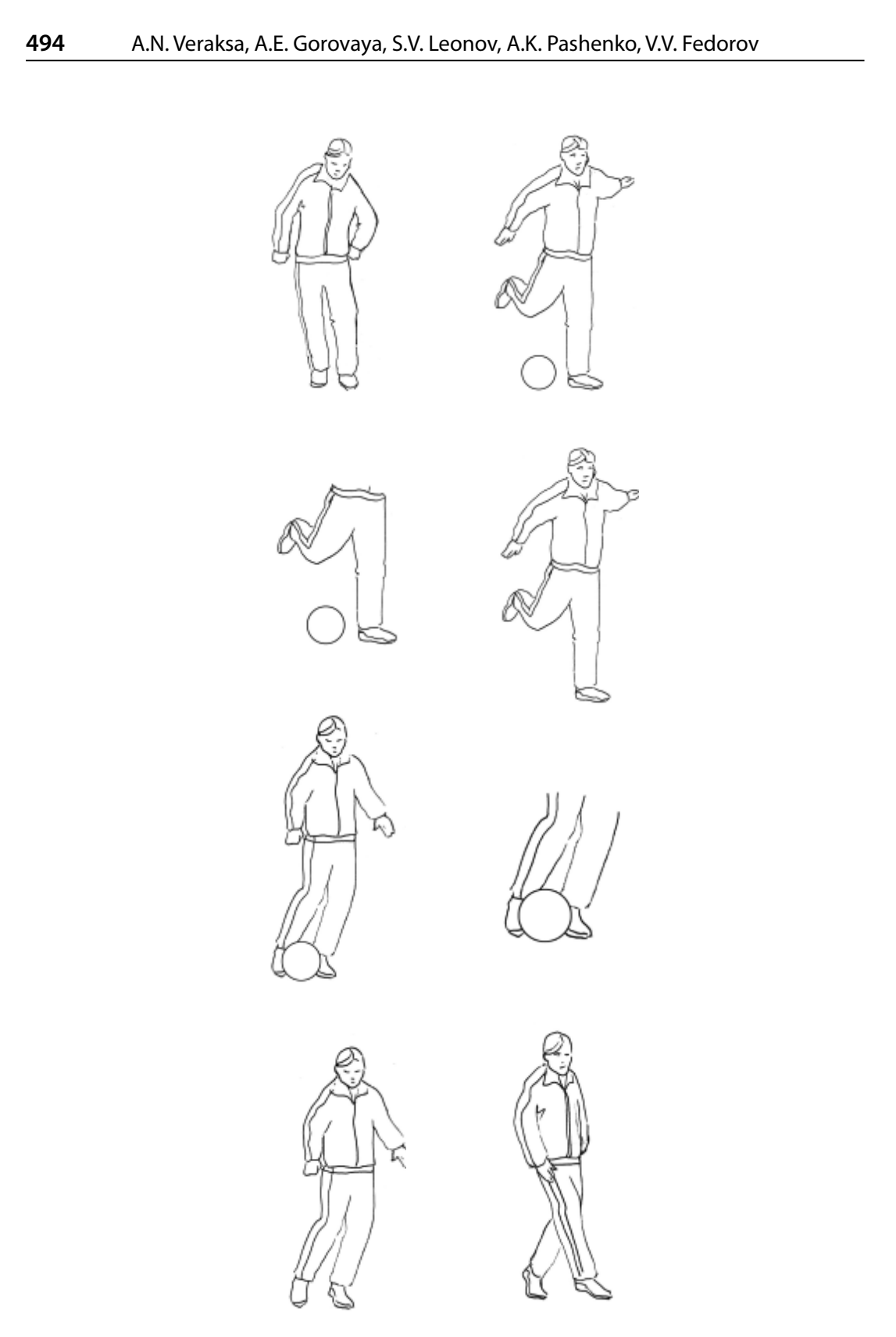$$
\text { 이 왕 }
$$$$
\text { 战 }
$$

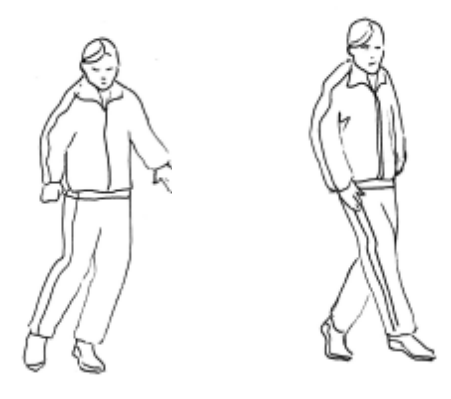


Shot-by-shot video images of the mini-movie for the metaphors group:
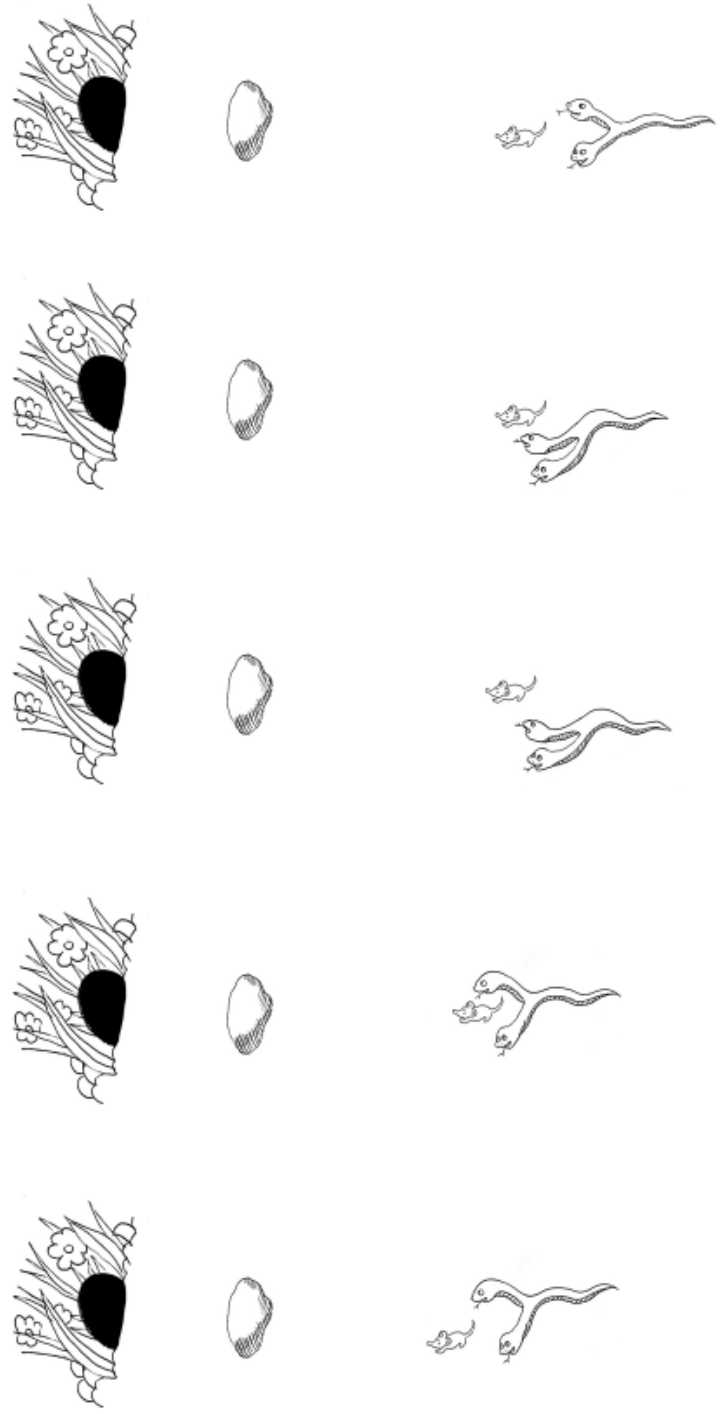

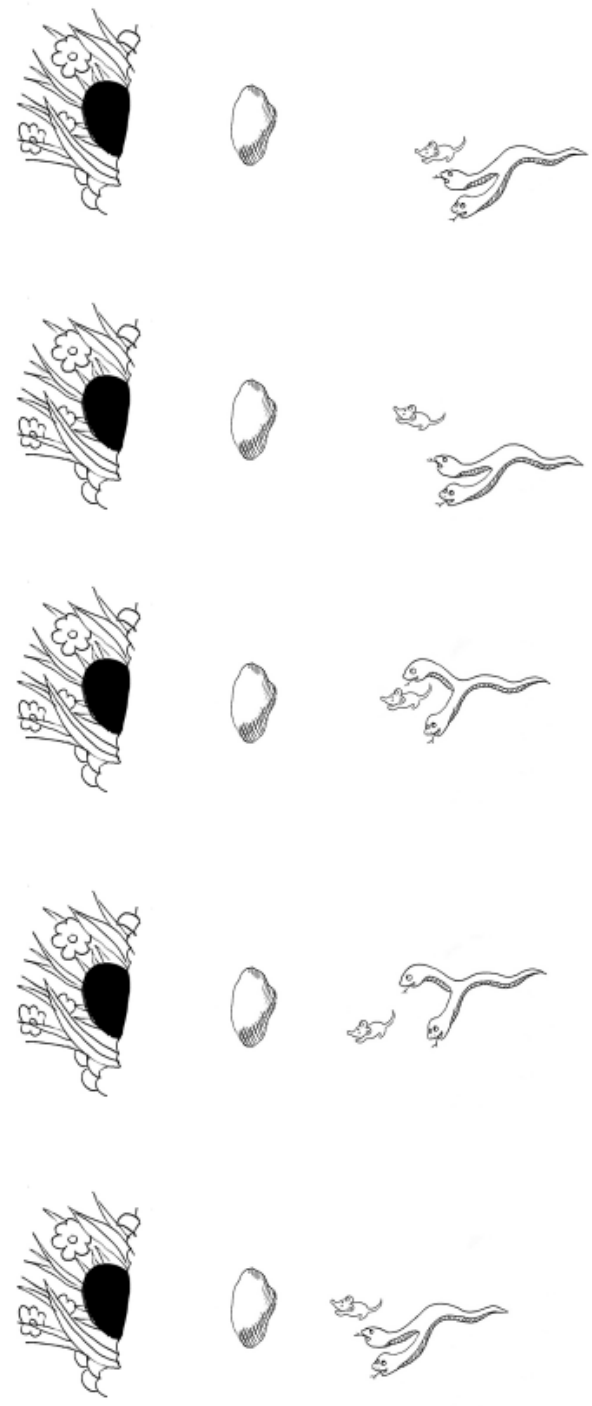

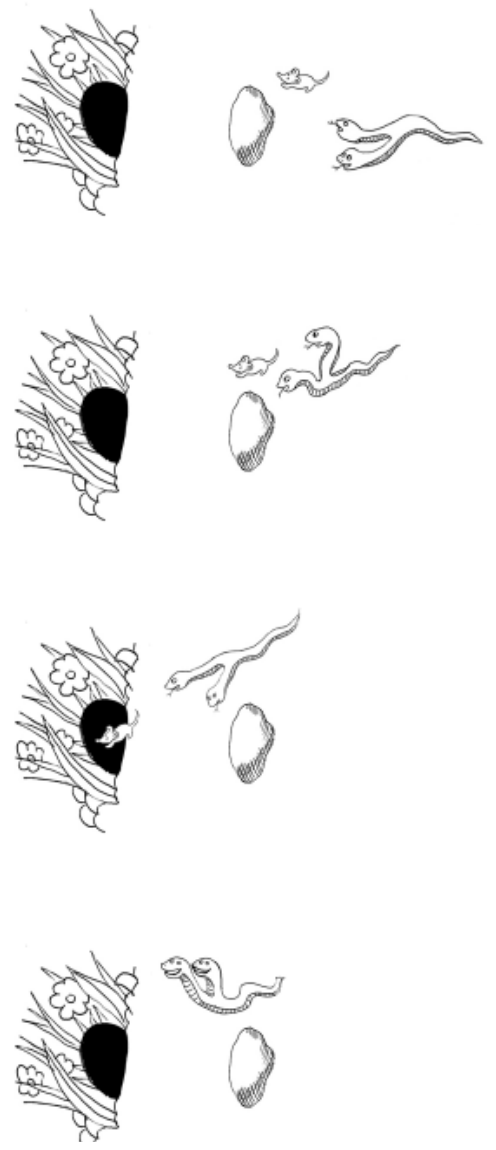